\title{
Inflation in an effective gravitational model and asymptotic safety
}

\author{
Lei-Hua Liu, ${ }^{1, *}$ Tomislav Prokopec, ${ }^{1, \dagger}$ and Alexei A. Starobinsky ${ }^{2,3, *}$ \\ ${ }^{1}$ Institute for Theoretical Physics, Spinoza Institute and the Center for Extreme Matter and Emergent \\ Phenomena (EMMEФ), Utrecht University, Buys Ballot Building, Princetonplein 5, \\ 3584 CC Utrecht, Netherlands \\ ${ }^{2}$ L. D. Landau Institute for Theoretical Physics RAS, Moscow 117334, Russia (Russian Federation) \\ ${ }^{3}$ Kazan Federal University, Kazan 420008, Republic of Tatarstan, Russia (Russian Federation)
}

(Received 18 June 2018; published 6 August 2018)

\begin{abstract}
We consider an inflationary model motivated by quantum effects of gravitational and matter fields near the Planck scale. Our Lagrangian is a resummed version of the effective Lagrangian recently obtained by Demmel, Saueressig, and Zanusso [A proper fixed functional for four-dimensional quantum Einstein gravity, J. High Energy Phys. 08 (2015) 113.] in the context of gravity as an asymptotically safe theory. It represents a refined Starobinsky model, $\mathcal{L}_{\text {eff }}=M_{\mathrm{P}}^{2} R / 2+(a / 2) R^{2} /\left[1+b \ln \left(R / \mu^{2}\right)\right]$, where $R$ is the Ricci scalar, $a$ and $b$ are constants, and $\mu$ is an energy scale. By implementing the COBE normalization and the Planck constraint on the scalar spectrum, we show that increasing $b$ leads to an increased value of both the scalar spectral index $n_{s}$ and the tensor-to-scalar ratio $r$. Requiring $n_{s}$ to be consistent with the Planck Collaboration upper limit, we find that $r$ can be as large as $r \simeq 0.01$, the value possibly measurable by Stage IV CMB ground experiments and certainly from future dedicated space missions. The predicted running of the scalar spectral index $\alpha=d n_{s} / d \ln (k)$ is still of the order $-5 \times 10^{-4}$ (as in the Starobinsky model), about 1 order of magnitude smaller than the current observational bound.
\end{abstract}

DOI: $10.1103 /$ PhysRevD.98.043505

\section{INTRODUCTION}

The $\Lambda$ CDM model supplemented with inflation is currently the best paradigm that provides a consistent quantitative description for the accelerating expansion of the Universe, cold dark nonbaryonic matter (CDM), the origin of large scale structure (LSS) and temperature fluctuations in the cosmic microwave background (CMB). In addition, cosmic inflation [1-4] provides an elegant and minimal solution of the horizon, flatness and homogeneity problems and can dilute magnetic monopoles if they were produced before inflation. In most inflationary models, a scalar fielddubbed an "inflaton"- is invoked to drive inflation and to seed primordial inhomogeneous adiabatic scalar perturbations measured by observations at present. The inflaton can be a new scalar field that couples weakly to the Standard Model fields. (Such a coupling - be it direct or indirect-is required for successful postinflationary stage of the energy transfer from inflaton to other quantum fields, (pre)heating of matter and radiation and transition to the standard hot big bang.) Or it can be embedded into a (grand-unified) extension of the standard model, such as a Higgs-like field required for mass generation.

\footnotetext{
*L.Liu1@uu.nl t.prokopec@uu.nl

¥alstar@landau.ac.ru
}

A notable alternative are purely geometric effective scalar degrees of freedom-in this work, we shall call these fields "scalarons"- which can be generated by, e.g., quantum-gravitational effects. Namely, generically quantum fluctuations of matter and gravitational fields at the Planck scale generate higher-derivative local gravitational operators in the effective action. If these operators are of the form of a function of the Ricci scalar $R, f(R)$, by the method of Lagrange multipliers one can introduce a scalaron field which can play the role of the inflaton. Donoghue [5] pointed out that it is natural to expect that at energies much below the Planck scale only one of those higher-dimensional operators plays a significant role for the Universe's dynamics. The simplest such model was constructed already in 1980 by Starobinsky [2], and its predictions agree very well with current observations [6]. The Lagrangian density in this model is of the form, $\mathcal{L}=\left(M_{\mathrm{P}}^{2} / 2\right) R+a R^{2} / 2$ where $a \approx 10^{9} \gg 1$ and $M_{\mathrm{P}}=1 / \sqrt{8 \pi G} \simeq 2.45 \times 10^{18} \mathrm{GeV}$, up to small one-loop corrections from matter quantum fields which are responsible, in particular, for the scalaron decay and creation of all standard matter after the end of inflation, see [7] for a more detailed quantitative description of the latter processes. The latter corrections which are of the type $\mathcal{R} \mathcal{R} \ln (\mathcal{R} \mathcal{R})$ at large curvatures, where $\mathcal{R} \mathcal{R}$ denotes some scalar quadratic combination built from the Riemann tensor, follow from perturbative quantum gravity [8-10], from the effective 
field theory approach to quantum gravity (conserving the local Lorentz invariance and general covariance) [11], from the calculation of a renormalized average value of the energy-momentum tensor of quantum matter fields in external gravitational fields [12], as well as by modern developments in this area. However, once one-loop corrections have been taken into account, it is natural to think about higher-loop ones and try to account for them in some approximation. That is why in this paper we considered a refined Starobinsky model which is based on some assumption about the form of re-summed logarithmic multi-loop corrections to the $R^{2}$ term.

While it is well known that general relativity theory is nonrenormalizable [13-15], this is not so for the so-called fourth-order gravity which contains terms $R^{2}$ and $W_{\alpha \beta \gamma \delta} W^{\alpha \beta \gamma \delta}$, where $W_{\alpha \beta \gamma \delta}$ is the Weyl tensor, in the Lagrangian density in addition to the Einstein term $R$. The fact that coefficients in front of these new terms are dimensionless (in particular, the $a$ coefficient in front of the $R^{2}$ term) already suggests that this theory may be renormalizable in some sense, e.g., by power counting. However, it has a ghost in the tensor sector (though not in the scalar sector). Weinberg has proposed that gravity may be renormalizable in a weaker sense, which goes under the name of asymptotic safety [16-19]. If gravity is an asymptotically safe theory, its ultraviolet completion would possess a finite number of relevant operators, and the corresponding coupling constants could be (in principle) determined by a finite number of measurements, thereby making it predictable. Initially, Weinberg proposed [17] that one could use renormalization group methods in a small $\epsilon$ expansion around $D=2$, i.e., $D=2+\epsilon$, around $D=4$, but soon recognized that is not a very good expansion near $\epsilon \simeq 2$. A modern approach to study the ultraviolet sector of quantum gravity uses functional renormalization group methods [20] (for a review see [21]) and the results support the asymptotic safety hypothesis. Recently, by working within the framework of asymptotic safety, Demmel, Saueressig, and Zanusso [22] have assumed that quantum gravity in the ultraviolet may be represented by a series of local operators, starting with $R, R^{2}$, etc. and they have shown that the coefficient of $R^{2}$ runs approximately logarithmically with $R$. This then implies that-after resummation under some suitable assumptions-at low energies, the effective theory Lagrangian can be represented by

$$
\mathcal{L} \simeq \frac{M_{\mathrm{P}}^{2}}{2} R+\frac{a R^{2}}{2\left[1+b \ln \left(R / \mu^{2}\right)\right]},
$$

where $\mu$ is a renormalization scale and $a=a(\mu) \gg 1$ and $b=b(\mu)$ are $\mu$-dependent constants; i.e., once $\mu$ is fixed, the values of $a$ and $b$ are also fixed. Since the Hubble (curvature) scale during the observable part of inflation (last 50-60 e-foldings) is much below the Planck scale, $H_{I} \sim M_{P} / \sqrt{a} \sim 10^{14} \mathrm{GeV} \ll E_{\mathrm{P}} \simeq 1.2 \times 10^{19} \mathrm{GeV}$, it is reasonable to expect that the model Lagrangian (1) represents reasonably well the true effective theory of modified gravity at inflationary curvatures. Furthermore, we expect that predictions of the inflationary model driven by the effective Lagrangian (1) do not differ by much from the predictions of the Starobinsky model. The analysis conducted in this work confirms that expectation, and moreover we provide an accurate answer to the question: in precisely what way predictions of the model (1) differ from those of the Starobinsky model.

It is very important that these quantum-gravitational corrections, though being small compared to the bare $a R^{2} / 2$ term, can still be important both for dynamics of inflation and generation of perturbations, thus they can be observable. The reason for this is that the pure $\mathcal{L}=a R^{2} / 2$ theory admits exact de Sitter solutions with any curvature. As a result, slow roll of curvature during inflation and the final graceful exit from it, as well as the slope of the scalar perturbation spectrum $n_{s}$ and the tensor-to-scalar ratio $r$, are governed by small corrections to this theory, namely, by the Einstein term $\propto R$ in the Starobinsky $R+R^{2}$ model which is much less than the $R^{2}$ term during inflation. Thus, quantum corrections in the model (1) have to be compared to this small term, too.

Of course, there is one more operator of canonical dimension four that can be added to (1), and that is $\Delta \mathcal{L}_{W W}=a_{W W} W_{\alpha \beta \gamma \delta} W^{\alpha \beta \gamma \delta}$. However, to avoid problems with the ghost in the tensor sector [23], in this work we assume that $a$ in (1) is as anomalously large as required by observations (in fact, by the smallness of large-scale inhomogeneous perturbations in the Universe). On the other hand, there is no theoretical or observational reasons to assume such a large value for $a_{W W}$, so $a_{W W} \ll a$. As a result, the $W W$ term can be neglected during the observable part of inflation. (One may think that $R_{\mu \nu} R^{\mu \nu}$ should also be considered, but that term is expressible in terms of the Gauss-Bonnet term which does not contribute to the equations of motion in four spacetime dimensions, and hence need not be considered separately.) Furthermore, there are higher-dimensional operators of the form $a_{n} R^{n} / M_{\mathrm{P}}^{2(n-2)}(n \geq 3)$, etc., but if none of $a_{n}$ 's is anomalously large, their contribution will be unimportant during inflation, and hence can be neglected. Indeed, a rough estimate can be made as follows. During the $R+R^{2}$ inflation, $R / M_{\mathrm{P}}^{2} \approx 2 N /(3 a) \ll 1$, where $N$ is the number of $e$-foldings from the end of inflation $(1 \ll N \lesssim 60)$, and hence the relative contribution of these terms compared to the nonleading Einstein term $M_{P}^{2} R / 2$, as explained above, is $\sim a_{n}\left(R / M_{\mathrm{P}}^{2}\right)^{n-1} \sim a_{n}(N / a)^{n-1} \ll 1(\forall n \geq 3)$ as far as $N a_{n}^{1 /(n-1)} \ll a$, see also [24] in this connection. Thus, as the above argument suggests, these models do not suffer from large corrections coming from higher-dimensional operators. However, with a suitable amount of fine tuning, it is possible to do away with lower-dimensional operators, 
such that one still gets a viable inflationary model driven by higher-dimensional operators of dimension four and higher [25].

We are not the first who consider an effective gravity inspired by asymptotic safety to drive inflation. Notable initial attempts are due to Bonanno, Reuter and Saueressig [26-29] and more recently by Falls et al. [30]. Except in the most recent Ref. [30], these works used a time-dependent cutoff which breaks general covariance and therefore require a better justification. More recently, Refs. [31-33] have renewed the idea that inflation may be driven within an effective quantum gravity inspired by asymptotic safety, see also the recent review [34]. In contrast to our model, in these works inflation is driven by a (scale-dependent) cosmological constant, $\Lambda=\Lambda(\mu)$, and the authors do not explain how inflation ends. While our effective model takes into account the running of the coupling constants, and in that respect it is motivated by the recent results on asymptotically safe gravity theories [22], rigorously speaking it is not an asymptotically safe gravity model of inflation where inflation occurs close to the conformal point. Instead, in our model inflation occurs in the infrared regime rather far from the ultraviolet fixed point. Furthermore, the cosmological constant is in our model assumed to be fine tuned to zero and inflation is driven by the $a(\mu, R) R^{2}$-term, such that graceful exit problem is naturally solved. A sufficiently long lasting inflation is obtained by assuming an anomalously large coefficient $a$, which is consistent with the renormalization group equations, since $a$ appears as an integration constant [22]. Other notable papers that discuss inflation in effective theories inspired by quantum gravitational effects which, like us, study inflationary models inspired by quantum corrections to the Starobinsky model, include [35-40]. Similar effective models can also arise from reconstruction of $f(R)$ gravity from observations [41].

This paper is organized as follows. In Sec. II, we present our inflationary model. After the background equations of motion are introduced in II A, in II B we discuss the specifics of cosmological perturbations in our model and in II C we discuss how to implement the COBE constraint. In Sec. III, we present our main results, which include the dependence of the scalar spectral index $n_{s}$, its running $\alpha$ and the tensor-to-scalar ratio $r$ on the parameter $b$ in Eq. (1). Finally, we conclude in Sec. IV. In the Appendix, an alternative derivation of $n_{s}$ and $r$ directly in the Jordan frame is presented.

Here we adopt units in which the speed of light $c=1$ and the reduced Planck constant $\hbar \equiv h /(2 \pi)=1$.

\section{THE MODEL}

In a recent paper, Demmel, Saueressig, and Zanusso [22] have considered quantum gravitational corrections to Einstein general relativity and they found that at highenergy scales quantum gravitational effects generate a contribution to the effective action of the form $\Delta S_{\text {eff }}=$ $\int d^{4} x a(\mu) R^{2} / 2$, where $a$ is a parameter that slowly (logarithmically) varies with scale. By dimensional transmutation [42], one can argue that, since $a(\mu)$ is dimensionless, it must be a function of a dimensionless quantity $R / \mu^{2}$, i.e., $a=a\left(R / \mu^{2}\right)=a_{0}\left[1-b_{0} \ln \left(R / \mu^{2}\right)\right]$, where $R$ is the Ricci scalar. While $a_{0}=a\left(R / \mu^{2}=1\right)$ is a free constant (to be fixed by measurements), Ref. [22] found that,

$$
b_{0}=\frac{40}{55296 \pi^{2} a_{0}+95} .
$$

In this work, we assume that, if a suitable renormalization group (RG) resummation is made, one gets an improved effective action of the form

$S=\int d^{4} x \sqrt{-g} \frac{1}{2}\left[M_{\mathrm{P}}^{2} R+\frac{a R^{2}}{1+b \ln \left(R / \mu^{2}\right)}+\mathcal{O}\left(R^{3}\right)\right]$,

where $M_{\mathrm{P}}=(8 \pi G)^{-1 / 2}$ is the reduced Plank mass, $a$ and $b$ are positive constants and $a \gg 1$. For notational simplicity we have dropped the subscript 0 on parameters $a$ and $b$ in (3). While $a$ is a free constant to be fixed by measurements, $b$ receives contributions both from quantum effects of matter and gravitational fields that are calculable by perturbative methods within a given theory and from threshold effects from the (unknown) Planck scale physics. In this work, we assume that the action (3) drives inflation. According to the COBE normalization of scalar cosmological perturbations, the Hubble rate $H$ during the observable part of inflation, $H \sim H_{\mathrm{I}} \sim 10^{14} \mathrm{GeV}$, is much smaller than the Planck energy, $E_{\mathrm{P}} \simeq 1.2 \times 10^{19} \mathrm{GeV}$. For that reason we expect that the higher-dimensional operators $\mathcal{O}\left(R^{3}\right)$ in (3) present both a negligible contribution to evolution of the inflaton and to measurable properties of cosmological perturbations in our model and, therefore, we neglect these higher-order terms in the remainder of this work. While in pure gravity $a$ and $b$ are related by Eq. (2), adding matter may change that relation [43], and hence we shall relax that condition. On the other hand, if the action (3) is to be used as a model of inflation, then the amplitude of scalar cosmological perturbations is fixed by the COBE normalization. This fixes one relation between $a$ and $b$ in (3), such that ultimately our inflationary model has one free parameter. We shall study how observable predictions of our model depend on that free parameter.

Note that in the limit of small $b$, the second term in the effective action (3) appears to be equivalent to (a) $a R^{2}-a b R^{2} \ln \left(R / \mu^{2}\right)$ from Ref. [22] as well as to (b) $a \mu^{4}\left(R / \mu^{2}\right)^{2-b}$. Inflation built based on the former form was also studied in $[39,41,44]$, while on the latter form-in [37,45-47]. One should point out, however, that for any finite $b$ these models are not equivalent to (3). Note also the crucial difference of our model from that considered in [48] where the first (Einstein) term in the action (3) was absent. 
As explained above, this term, though being small compared to the second one during inflation, strongly affects slow roll of $R$ and the values of $n_{s}$ and $r$. In addition, it provides a graceful exit from inflation.

Let us now proceed to analyze inflation governed by (3). The action (3) is equivalent to,

$$
S=\int d^{4} x \sqrt{-g} \frac{1}{2}\left[f(\Phi)+\omega^{2}(R-\Phi)\right],
$$

where $f(\Phi)=M_{\mathrm{P}}^{2} \Phi+a \Phi^{2} /\left[1+b \ln \left(\Phi / \mu^{2}\right)\right], \Phi$ is a real scalar field (dubbed "scalaron" in [2]) and $\omega=\omega(x)$ is a Lagrange multiplier (constraint) field (whose equation of motion imposes $\Phi=R$ ). Now upon varying the action (3) with respect to $\Phi$ and solving the resulting equation, one obtains

$$
f^{\prime}(\Phi)-\omega^{2}=0
$$

where

$$
f^{\prime}(\Phi)=\frac{d f}{d \Phi} \equiv F(\Phi)
$$

Inserting (5) into (3) results in an action that is on-shell equivalent to (3),

$$
S=\int d^{4} x \sqrt{-g} \frac{1}{2}[f(\Phi)+F(\Phi)(R-\Phi)]
$$

Note that the scalaron $\Phi$ in (7) is nonminimally coupled to gravity via the term $F(\Phi) R / 2$. It is hence useful to refer to this form of the action as the Jordan frame. It is well known that one can transform (7) to the Einstein frame through a suitable conformal transformation $g_{\mu \nu}=\Omega^{2}(x) g_{\mu \nu}^{E}$, where $\Omega=\Omega(x)$ is some still-to-be-specified local function. By making use of the standard conformal transformation for the Ricci scalar and metric determinant $g$ one obtains,

$$
\begin{aligned}
S= & \int d^{4} x \sqrt{-g_{E}} \frac{1}{2}\left[\Omega^{2} F(\Phi)\left(R_{E}-6 g_{E}^{\mu \nu} \frac{\left(\nabla_{\mu}^{E} \nabla_{\nu}^{E} \Omega\right)}{\Omega}\right)\right. \\
& \left.-\Omega^{4}(F(\Phi) \Phi-f(\Phi))\right] .
\end{aligned}
$$

By choosing the conformal function according to,

$$
\Omega^{2}(\Phi)=\frac{M_{\mathrm{P}}^{2}}{F(\Phi)}
$$

partially integrating the second term in the first line of Eq. (8) and dropping the resulting boundary term, the action of (8) becomes,

$$
\begin{aligned}
S= & \int d^{4} x \sqrt{-g_{E}}\left[\frac{M_{\mathrm{P}}^{2}}{2} R_{E}-3 M_{\mathrm{P}}^{2} g_{E}^{\mu \nu} \frac{\left(\nabla_{\mu}^{E} \Omega\right)\left(\nabla_{\nu}^{E} \Omega\right)}{\Omega^{2}}\right. \\
& \left.-\frac{1}{2} \Omega^{4}(F \Phi-f)\right],
\end{aligned}
$$

where $\Omega=\Omega(\Phi)$ is given by (9). We have gotten rid of the higher-dimensional gravitational operator, but the prize is the emergence of a dynamical scalar field-the scalaron field. Note that the scalaron has a noncanonical kinetic term, however, and one can bring it to a canonical form by the following transformation to the Einstein frame,

$$
\phi_{E}=-\frac{M_{P}}{2} \sqrt{6} \ln \left(\Omega^{2}(\Phi)\right),
$$

where the field mapping is such that $\phi_{E}=0$ when $\Omega=1$. Notice that instead of (11) one could have chosen a field transformation with the opposite sign. In fact, that transformation is equivalent to (11) in the sense that the resulting Einstein frame potential would be the mirror image around $\phi_{E}=0$ of the potential obtained by the transformation (11). When (11) is exacted one obtains the following Einstein frame action,

$S=\int d^{4} x \sqrt{-g_{E}}\left[\frac{M_{\mathrm{P}}^{2}}{2} R_{E}-\frac{1}{2} g_{E}^{\mu \nu}\left(\partial_{\mu} \phi_{E}\right)\left(\partial_{\nu} \phi_{E}\right)-V_{E}\left(\phi_{E}\right)\right]$,

where $V_{E}\left(\phi_{E}\right)$ denotes the Einstein frame potential,

$$
V_{E}\left(\phi_{E}\right)=\frac{M_{P}^{4}}{2} \frac{F \Phi-f}{F^{2}},
$$

and where, in light of Eqs. (9), (11) and (6),

$$
\begin{aligned}
F\left(\phi_{E}\right) & =M_{\mathrm{P}}^{2} \exp \left(\sqrt{\frac{2}{3}} \frac{\phi_{E}}{M_{\mathrm{P}}}\right) \\
& =M_{\mathrm{P}}^{2}+\frac{a \Phi\left[2-b+2 b \ln \left(\Phi / \mu^{2}\right)\right]}{\left[1+b \ln \left(\Phi / \mu^{2}\right)\right]^{2}} .
\end{aligned}
$$

This equation defines the frame transformation, $\phi_{E}=\phi_{E}(\Phi)$. Unfortunately, its inverse $\Phi=\Phi\left(\phi_{E}\right)$ is not a simple function that can be written in a closed form, so we write $V_{E}\left(\phi_{E}\right)$ as a function of $\Phi$, but we keep in mind that $\Phi$ can be expressed in terms of $\phi_{E}$ via (14).

Taking the point of view that gravity is an effective field theory [5], the theory (12) can be (canonically) quantized. There are two dynamical fields in (12): the inflaton $\phi_{E}$ and the graviton $g_{\mu \nu}$ that ought to be quantized. In what follows, we first discuss the dynamics of classical fields (condensates) and then the (tree level) dynamics of quantum perturbations. 


\section{A. Background dynamics and properties of cosmological perturbations}

The action (12) can be used to drive inflation, provided the quantum field $\hat{\phi}_{E}$ develops a large expectation value. If the field is approximately homogeneous with respect to a space-like hypersurface, then it can be decomposed into its condensate (inflaton) and small perturbations as follows,

$$
\begin{aligned}
\hat{\phi}_{E}(x) & =\phi_{E 0}(t)+\hat{\varphi}_{E}(x), \\
\phi_{E 0}(t) & =\left\langle\hat{\phi}_{E}(x)\right\rangle \equiv \operatorname{Tr}\left[\hat{\rho}(t) \hat{\phi}_{E}(x)\right],
\end{aligned}
$$

where $\hat{\rho}(t)$ denotes the density operator. Similarly, the metric tensor (in Einstein frame) can be written as

$\hat{g}_{\mu \nu}^{E}(x)=g_{\mu \nu}^{E b}(t)+\delta \hat{g}_{\mu \nu}^{E}(x)$,

$g_{\mu \nu}^{E b}(t)=\left\langle\hat{g}_{\mu \nu}^{E}(x)\right\rangle=\operatorname{diag}\left(-1, a_{E}^{2}(t), a_{E}^{2}(t), a_{E}^{2}(t)\right)$,

and $\delta \hat{g}_{\mu \nu}^{E}(x)=a_{E}^{2}(t) \hat{h}_{\mu \nu}(x)$, where $a_{E}=a_{E}(t)$ is the Einstein frame scale factor and $\hat{h}_{\mu \nu}(x)$ is the (suitably rescaled) graviton perturbation in Einstein frame. We work here in the traceless transverse (Lifshitz) gauge, in which $\hat{h}_{0 \mu}=0$, $\partial_{i} \hat{h}_{i j}(x)=0=\hat{h}_{i i}(x)$.

The dynamics of the inflaton condensate is governed by the equation of motion,

$$
\ddot{\phi}_{E 0}(t)+3 H \dot{\phi}_{E 0}(t)+\frac{d V_{E}}{d \phi_{E 0}}=0
$$

where we neglected any backreaction from quantum fluctuations. Analogously, evolution of the background geometry is governed by the Friedmann (or FLRW) equations,

$$
\begin{gathered}
H_{E}^{2} \equiv\left(\frac{\dot{a}_{E}}{a_{E}}\right)^{2}=\frac{1}{3 M_{\mathrm{P}}^{2}}\left(\frac{\dot{\phi}_{E 0}^{2}}{2}+V_{E}\left(\phi_{E 0}\right)\right) \\
\dot{H}_{E}=-\frac{\dot{\phi}_{E 0}^{2}}{2 M_{\mathrm{P}}^{2}},
\end{gathered}
$$

where $H_{E}$ is the Hubble parameter in Einstein frame and $\dot{H}_{E}=d H_{E} / d t$.

Let us now consider scalar and tensor cosmological perturbations in the model (12). It is convenient to decompose scalar and graviton perturbations in Fourier modes,

$$
\begin{aligned}
\hat{\varphi}_{E}(t, \vec{x})= & \int \frac{d^{3} k}{(2 \pi)^{3}} \mathrm{e}^{\vec{k} \cdot \vec{x}}\left[\varphi(t, k) \hat{a}(\vec{k})+\varphi^{*}(t, k) \hat{a}^{\dagger}(-\vec{k})\right] \\
\hat{h}_{i j}(t, \vec{x})= & \sum_{\alpha=+, \times} \int \frac{d^{3} k}{(2 \pi)^{3}} \mathrm{e}^{\vec{k} \cdot \vec{x}}\left[\epsilon_{i j}^{\alpha}(\vec{k}) h(t, k) \hat{b}_{\alpha}(\vec{k})\right. \\
& \left.+\epsilon_{i j}^{\alpha}(-\vec{k})^{*} h^{*}(t, k) \hat{b}_{\alpha}^{\dagger}(-\vec{k})\right]
\end{aligned}
$$

where $k=\|\vec{k}\|, \quad \hat{a}(\vec{k})$ and $\hat{a}^{\dagger}(\vec{k})$ the annihilation and creation operators for scalar perturbations, $\hat{a}(\vec{k})|\Omega\rangle=0$ annihilates the vacuum state $|\Omega\rangle$, and $\varphi(t, k)$ and $\varphi^{*}(t, k)$ are the two linearly independent solutions to the mode function equation,

$$
\left(\frac{d^{2}}{d t^{2}}+3 H_{E} \frac{d}{d t}+\frac{k^{2}}{a_{E}^{2}}+\frac{d^{2} V_{E}}{d \phi_{E 0}^{2}}\right) \varphi(t, k)=0 .
$$

$\epsilon_{i j}^{\alpha}(\vec{k})$ in (20) are the spin 2 polarization tensors, obeying $\sum_{\alpha} \epsilon_{i j}^{\alpha}(\vec{k}) \epsilon_{k l}^{\alpha}(-\vec{k})^{*}=\left(P_{i k} P_{j l}+P_{i l} P_{j k}-P_{i j} P_{k l}\right) / 2, \quad P_{i j}=$ $\delta_{i j}-k_{i} k_{j} / k^{2}, \sum_{i j} \epsilon_{i j}^{\beta}(\vec{k}) \epsilon_{i j}^{\alpha}(-\vec{k})^{*}=\delta_{\alpha \beta}, \hat{b}_{\alpha}(\vec{k})$ and $\hat{b}_{\alpha}^{\dagger}(\vec{k})$ are the graviton annihilation and creation operators $\left(\hat{b}_{\alpha=+, \times}(\vec{k})|\Omega\rangle=0\right)$ and $h(t, k)$ and $h^{*}(t, k)$ are the graviton mode functions that satisfy,

$$
\left(\frac{d^{2}}{d t^{2}}+3 H_{E} \frac{d}{d t}+\frac{k^{2}}{a_{E}^{2}}\right) h(t, k)=0
$$

In the zero curvature gauge (in which the spatial scalar graviton perturbation vanishes), scalar curvature perturbation is given by,

$$
\mathcal{R}(x)=-\frac{H_{E}}{\dot{\phi}_{E 0}} \varphi(x), \quad \dot{\phi}_{E 0}^{2}=2 \epsilon_{E} H_{E}^{2} M_{\mathrm{P}}^{2},
$$

where $\epsilon_{E}$ is defined in Eq. (37) below. Late time observers can measure properties of cosmological perturbations, which are characterized by the corresponding spectra, which are defined as

$$
\begin{aligned}
\left\langle\hat{\mathcal{R}}(t, \vec{x}) \hat{\mathcal{R}}\left(t, \vec{x}^{\prime}\right)\right\rangle & =\int \frac{d k}{k} \mathrm{e}^{i \vec{k} \cdot\left(\vec{x}-\vec{x}^{\prime}\right)} \Delta_{R}^{2}(t, k) \\
\left\langle\hat{h}_{i j}(t, \vec{x}) \hat{h}_{i j}\left(t, \vec{x}^{\prime}\right)\right\rangle & =\int \frac{d k}{k} \mathrm{e}^{i \vec{k} \cdot\left(\vec{x}-\vec{x}^{\prime}\right)} \Delta_{t}^{2}(t, k)
\end{aligned}
$$

where during inflation the scalar and tensor spectrum can be calculated in terms of scalar and graviton mode functions as follows,

$$
\begin{aligned}
& \Delta_{R}^{2}(t, k) \equiv \Delta_{s}^{2}(t, k)=\frac{k^{3}}{8 \pi^{2} \epsilon_{E} M_{\mathrm{P}}^{2}}|\varphi(t, k)|^{2} \\
& \Delta_{t}^{2}(t, k)=\frac{2 k^{3}}{\pi^{2} M_{\mathrm{P}}^{2}}|h(t, k)|^{2}=16 \epsilon_{E} \Delta_{s}^{2},
\end{aligned}
$$

where we made use of Eqs. (20) and (23). When canonically normalized and to leading order in slow roll approximation, the mode functions on super-Hubble scales can be approximated by, $|\varphi|^{2} \simeq\left[H_{*}^{2} /\left(2 k^{3}\right)\right]\left[k /(a H)_{*}\right]^{n_{s}-1}$; an analogous approximation holds for the graviton mode functions, $|h|^{2} \simeq\left[H_{*}^{2} /\left(2 k^{3}\right)\right]\left[k /(a H)_{*}\right]^{n_{t}}$. 
Astronomers usually parametrize the observed spectra as follows,

$$
\begin{aligned}
& \Delta_{s}^{2}(k)=\Delta_{s *}^{2}\left(\frac{k}{k_{*}}\right)^{n_{s}(k)-1} \\
& \Delta_{t}^{2}(k)=\Delta_{t *}^{2}\left(\frac{k}{k_{*}}\right)^{n_{t}(k)}
\end{aligned}
$$

where $k_{*}$ is a fiducial comoving momentum usually chosen to be $k_{*}=0.05(\mathrm{Mpc})^{-1}$ or $k_{*}=0.002(\mathrm{Mpc})^{-1}, \Delta_{s *}^{2} \equiv$ $A_{s}$ and $\Delta_{t *}^{2}$ are the amplitude of scalar and tensor spectra evaluated at $k=k_{*}$ and $n_{s}$ and $n_{t}$ are the scalar and tensor spectral indices, respectively. The COBE normalization [6] constrains

$$
\begin{aligned}
\ln \left(10^{10} A_{s}\right) & =3.089 \pm 0.036 \quad\left(\text { at } k_{*}=0.05 \mathrm{MeV}^{-1}\right) \\
& \Rightarrow A_{s}=(2.20 \pm 0.08) \times 10^{-9},
\end{aligned}
$$

while from theory of scalar inflationary perturbations we know that (to leading order in slow roll approximation),

$$
A_{s} \simeq \frac{H_{*}^{2}}{16 \pi^{2} \epsilon_{E^{*}} M_{\mathrm{P}}^{2}},
$$

where $H_{*}$ and $\epsilon_{E *}$ are the Hubble parameter and the principal slow roll parameter at the time when the perturbation with comoving momentum, $k=k_{*}$ crosses the Hubble radius (becomes super-Hubble) during inflation. Furthermore, observations constrain the scalar spectral index $n_{s}$ and the tensor-to-scalar ratio, defined by

$$
r\left(k=k_{*}=0.05 \mathrm{Mpc}^{-1}\right)=\frac{\Delta_{t *}^{2}}{\Delta_{s *}^{2}} .
$$

According to the Planck Collaboration [6], $n_{s}=0.9655 \pm 0.0062(68 \% \mathrm{CL}$, Planck TT + lowP,$\alpha=0)$,

when the running of the spectral index,

$$
\alpha \equiv\left[\frac{d n_{s}(k)}{d \ln (k)}\right]_{k=k_{*}},
$$

is fixed to zero. When the constraint on $\alpha$ is relaxed, however, the error bars on $n_{s}$ increase somewhat to become

$$
\begin{aligned}
n_{s} & =0.965 \pm 0.010 \quad(1 \sigma \text { error bars }) \quad \text { and } \\
\alpha & =-0.003 \pm 0.007 .
\end{aligned}
$$

When recent large scale structure (LSS) data are included [49], error bars on $n_{s}$ shrink and one finds preference for a negative running, $n_{s}=0.963 \pm 0.0045$ and $\alpha=-0.0104 \pm 0.0031$. However, these results are still to be confirmed. At this moment there are no measurements of tensor perturbations; instead the literature quotes upper bounds. For example, the joint analysis BICEP2/Keck and Planck data found [50] $r<0.12(95 \% \mathrm{CL})$ and more recently [51] the BICEP2/Keck Collaboration finds,

$r<0.09\left(95 \% \mathrm{CL}\right.$, at $\left.k_{*}=0.05 \mathrm{Mpc}^{-1}\right) \quad(\mathrm{BICEP} 2 / \mathrm{Keck})$,

while the more recent combined Planck, BICEP and Keck Array data [52] yield a tighter constraint, $r<0.064$.

\section{B. Cosmological perturbations in our model}

In what follows, we relate the observable parameters $n_{s}$, $r$ and $\alpha$ of scalar and tensor cosmological perturbations (27) to our model in Einstein frame, defined by Eqs. (12)-(14). For a later use, we note that the Einstein frame potential (13) can be written explicitly as

$$
V_{E}\left(\phi_{E}\right)=\frac{M_{\mathrm{P}}^{4}}{2} \frac{a \Phi^{2}\left(1+b \ln \left[\frac{\Phi}{\mu^{2}}\right]\right)^{2}\left(1+b \ln \left[\frac{\Phi}{e \mu^{2}}\right]\right)}{\left\{M_{\mathrm{P}}^{2}\left(1+b \ln \left[\frac{\Phi}{\mu^{2}}\right]\right)^{2}+2 a \Phi\left(1+b \ln \left[\frac{\Phi}{\sqrt{e} \mu^{2}}\right]\right)\right\}^{2}}
$$

where $\Phi=\Phi\left(\phi_{E}\right)$ is given in Eq. (14) and $e \approx 2.81$. We shall also need $d \Phi / d \phi_{E}$, which is easily obtained by differentiating (14),

$$
\frac{\partial \Phi}{\partial \phi_{E}}=\sqrt{\frac{2}{3}} \frac{\left(1+b \ln \left[\frac{\Phi}{\mu^{2}}\right]\right)\left(\left(1+b \ln \left[\frac{\Phi}{\mu^{2}}\right]\right)^{2} M_{\mathrm{P}}^{2}+a \Phi\left(2-b+2 b \ln \left[\frac{\Phi}{\mu^{2}}\right]\right)\right)}{a M_{P}\left[\left(2-3 b+2 b^{2}\right)+b \ln \left[\frac{\Phi}{\mu^{2}}\right]\left(4-3 b+2 b \ln \left[\frac{\Phi}{\mu^{2}}\right]\right)\right]} .
$$

Most of inflationary models exhibit attractor behavior, which means that the physical parameters (such as the spectra) are expressible in terms of the inflaton amplitude alone (in an attractor, $\dot{\phi}_{E}$ is a function $\phi_{E}$, and in the attractor known as slow roll approximation, $\dot{\phi}_{E}$ and higherorder time derivatives of the field are small in a well defined sense). In what follows, we apply the slow roll attractor results to our model (12)-(14).

From the canonical quantization of scalar and tensor perturbations (20), (21), (22) and Eq. (26) one can show that when inflaton is in its attractor regime and when slow roll approximation applies, $n_{s}$ and $n_{t}$ can be 
expressed in terms of geometric slow roll parameters as follows,

$$
n_{s}=1-2 \epsilon_{E}-\eta_{E}, \quad n_{t}=-2 \epsilon_{E}, \quad \epsilon_{E}=-\frac{\dot{H}_{E}}{H_{E}^{2}}, \quad \eta_{E}=\frac{\dot{\epsilon}_{E}}{H_{E} \epsilon_{E}} .
$$

In general, the spectral index $n_{s}$ is a function of $k$, and its running $\alpha=d n_{s} / d \ln (k)$, can be expressed in terms of slow roll parameters as

$$
\alpha=-\eta_{E}\left(2 \epsilon_{E}+\xi_{E}\right), \quad \xi_{E} \equiv \frac{\dot{\eta}_{E}}{\eta_{E} H_{E}} .
$$

In general, $\alpha$ also depends on $k$. However, current observations are not precise enough for a detection of $\alpha$ and thus only upper limits on $|\alpha|$ are available. For that reason in this work by $\alpha$ we mean $\alpha\left(k_{*}\right)$.

One can express geometric slow roll parameters in terms of the more traditional slow roll parameters defined in terms of derivatives of the inflaton potential $V_{E}$. For example, we have

$$
\begin{aligned}
& \epsilon_{V}=\frac{M_{\mathrm{P}}^{2}}{2}\left(\frac{V_{E}^{\prime}}{V_{E}}\right)^{2}, \quad V_{E}^{\prime} \equiv \frac{d V_{E}}{d \phi_{E}}=\frac{d \Phi}{d \phi_{E}} \frac{d V_{E}}{d \Phi} \\
& \eta_{V}=M_{\mathrm{P}}^{2} \frac{V_{E}^{\prime \prime}}{V_{E}}, \quad V_{E}^{\prime \prime}=\left(\frac{d \Phi}{d \phi_{E}}\right)^{2} \frac{d^{2} V_{E}}{d \Phi^{2}}+\frac{d^{2} \Phi}{d \phi_{E}^{2}} \frac{d V_{E}}{d \Phi}, \\
& \xi_{V}^{2}=M_{\mathrm{P}}^{4} \frac{V_{E}^{\prime} V_{E}^{\prime \prime \prime}}{V_{E}^{2}}, \quad V_{E}^{\prime \prime \prime}=\left(\frac{d \Phi}{d \phi_{E}}\right)^{3} \frac{d^{3} V_{E}}{d \Phi^{3}}+3 \frac{d^{2} \Phi}{d \phi_{E}^{2}} \frac{d \Phi}{d \phi_{E}} \frac{d^{2} V_{E}}{d \Phi^{2}}+\frac{d^{3} \Phi}{d \phi_{E}^{3}} \frac{d V_{E}}{d \Phi},
\end{aligned}
$$

where $V_{E}\left(\phi_{E}\right)$ and $d \Phi / d \phi_{E}$ are given in (35)-(36). Together with $d \ln (k)=d \ln (a H)$ (which expresses the fact that the amplitude of perturbations in slow roll get frozen at super-Hubble scales) and the Friedmann equations (18)-(19), Eq. (39) imply

$$
\epsilon_{V}=\epsilon_{E}, \quad \eta_{E}=-4 \epsilon_{V}-2 \eta_{V},
$$

and hence

$$
n_{s}=1-6 \epsilon_{V}+2 \eta_{V}, \quad n_{t}=-2 \epsilon_{V} .
$$

Furthermore, one can show that,

$$
\begin{gathered}
r=16 \epsilon_{E}=16 \epsilon_{V}=-8 n_{t} \\
\alpha=16 \epsilon_{V} \eta_{V}-24 \epsilon_{V}^{2}-2 \xi_{V}^{2} .
\end{gathered}
$$

Equation (42) is known as the one field consistency relation and it can be used, e.g., to check whether inflation is driven by a single (inflaton) field.

Notice that, while $n_{s}, r$ and $n_{t}$ (41), (42) are of first order in slow roll parameters, the running of the spectral index $\alpha$ (43) is of second order in slow roll parameters, and hence it is expected to be smaller than $n_{s}, r$ and $n_{t}$. As we shall see below, this expectation is indeed borne out in our model.
Finally, a useful quantity to define is the number of $e$-foldings, which in Einstein frame and in slow roll approximation can be calculated as follows, ${ }^{1}$

$$
\begin{aligned}
N \approx N_{E} & =\int_{t}^{t_{e}} H_{E}\left(t^{\prime}\right) d t^{\prime}=\frac{1}{M_{\mathrm{P}}} \int_{\phi_{E}(t)}^{\phi_{E e}} \frac{d \phi_{E}^{\prime}}{\sqrt{2 \epsilon_{E}\left(\phi_{E}^{\prime}\right)}} \\
& =\frac{1}{M_{\mathrm{P}}} \int_{\Phi(t)}^{\Phi_{e}} \frac{d \phi_{E}\left(\Phi^{\prime}\right) / d \Phi^{\prime}}{\sqrt{2 \epsilon_{V}\left(\Phi^{\prime}\right)}} d \Phi^{\prime},
\end{aligned}
$$

where $t_{e}$ denotes the time at the end of inflation (at which $\left.\epsilon_{E}=1\right)$, and $\phi_{E e}=\phi_{E}\left(t_{e}\right), \Phi_{e}=\Phi\left(t_{e}\right)$. Now Eq. (44) tells us how the number of $e$-foldings depends on $\Phi$ (or

\footnotetext{
${ }^{1}$ The formula (44) calculates the number of $e$-foldings in the Einstein frame in the slow roll approximation. The more appropriate measure of cosmological time is the number of $e$-foldings in Jordan frame, since that is the original (physical) frame in which observations are made. There is a simple relation between the number of $e$-foldings in the two frames. From Eq. (9) and $g_{\mu \nu}=\Omega^{2} g_{\mu \nu}^{E}$ one sees that $\ln \left(a_{J}\right)=\ln \left(a_{E}\right)+\frac{1}{2} \ln \left(M_{\mathrm{P}}^{2} / F(\Phi)\right)$, such that the number of $e$-foldings in two frames is related as $N_{J}(t)=N_{E}(t)-\left.\frac{1}{2} \ln \left(F(\Phi) / M_{\mathrm{P}}^{2}\right)\right|_{\Phi_{e}} ^{\Phi(t)}$, where $\Phi_{e}$ denotes the value of $\Phi$ at the end of inflation. Numerical evaluation shows that the difference between $N_{J}$ and $N_{E}$ is at most a few percent, which means that results presented in terms of Einstein rather than Jordan frame number of $e$-foldings will differ by at most a few percent. Since the uncertainly in the number of $e$-foldings due to the unknown evolution of the postinflationary universe is anyway at the level of ten percent, we can use the Einstein frame number of $e$-foldings without introducing a significant new error. Nevertheless, the expression for the number of $e$-foldings in the Jordan frame $N_{J}$ is presented in the Appendix.
} 
equivalently $\phi_{E}$ ), while Eqs. (39)-(40), (41)-(43) relate $n_{s}$, $r$, and $\alpha$ to $\Phi$ (or equivalently $\phi_{E}$ ). When taken together, these two sets of (parametric) relations tell us how $n_{s}, r$ and $\alpha$ depend on $N$. Unfortunately, the form of the effective potential (35) is rather complicated such that we were unable to perform the integral (44). Nevertheless, with a help of the symbolic package Mathematica, we were able to plot the relevant curves.

To summarize, in this section, we have shown how to parametrically express measurable quantities $n_{s}, r=-8 n_{t}$ and $\alpha$ in terms of the number of $e$-foldings $N$. In the next sub-section, we show how to implement the existing observational constraints, which primarily pose a restriction on the number of $e$-foldings and the amplitude of scalar cosmological perturbations.

\section{Implementing constraints}

Apart from the (obvious) constraint on the scalar spectral index, there are two principal constraints that we ought to impose on our inflationary model:

(1) the amplitude of the scalar spectrum, also known as the COBE constraint (29);

(2) the number of $e$-foldings $N$ (44).

The COBE constraint is given in Eq. (28), from which we can infer the value of the potential at the moment when the fiducial comoving momentum $k_{*}$ crosses the Hubble scale during inflation, $k_{*}=(a H)_{*}$ as follows,

$\frac{V_{E}\left(\phi_{E *}\right)}{M_{\mathrm{P}}^{4}}=48 \pi^{2} \epsilon_{E *} A_{s} \simeq 3 \times 10^{-10}\left(\frac{r_{*}}{5 \times 10^{-3}}\right)\left(\frac{A_{s}}{2.2 \times 10^{-9}}\right)$.

The constraint on the number of $e$-foldings $N$ is not watertight, as it hangs on "reasonable assumptions" on postinflationary evolution. Assuming e.g., (a) that evolution of the Hubble parameter during inflation is given, (b) that the scale of inflation is given and (c) that the Universe after inflation quite quickly (within one expansion time) reaches radiation era scaling, then one can rather accurately estimate the number of $e$-foldings at which observable scales cross the Hubble radius during inflation. However, there are no data that would unambiguously fix the scale of inflation, or the precise evolution of the Hubble parameter during or after inflation. To incorporate this uncertainty, usually one plots physical parameters for several values of $N$. In this work, we take the reasonable range of $N$ to be $N \in[50,65]$.

In the analysis of our inflationary model presented in Sec. II B, we enforce the COBE constraint as shown in (45), and we show results for $N=50$ and $N=65$. Our model (3) contains two free parameters $a$ and $b$ which determine the scale of inflation $H_{E *}$ at $N \in[50,65]$. Imposing the $\mathrm{COBE}$ constraint fixes one relation between $a$ and $b$, leaving one free parameter. Our results are shown as a function of that free parameter, which for definiteness we choose to be $b$, while $a=a(b)$.

To get an impression on how our potential looks in the Einstein frame, in Fig. 1 we show the potential $V_{E}=V_{E}(\Phi)$ defined in Eq. (35), where (at scale $\mu=10^{-5} \simeq H_{E *}$ ) the values of the couplings are, $a=7 \times 10^{8}$ and $b=10^{-2}$ (top curve), $b=10^{-3}$ (central curve) and $b=10^{-4}$ (bottom curve). For convenience we shall also use $\tilde{a}$ defined by, $a=10^{10} \tilde{a}$. As $b$ decreases and for large values of $\Phi$ the potential becomes flatter and flatter. In the limit when $b \rightarrow 0$ (Starobinsky inflation) and as $\Phi \rightarrow \infty$, the potential becomes exactly flat.

Figure 2 shows the allowed range of parameters $\tilde{a}=$ $10^{-10} a$ and $b$ for which one gets $N$ in the range $[50,65]$ (the COBE constraint (45) is imposed).

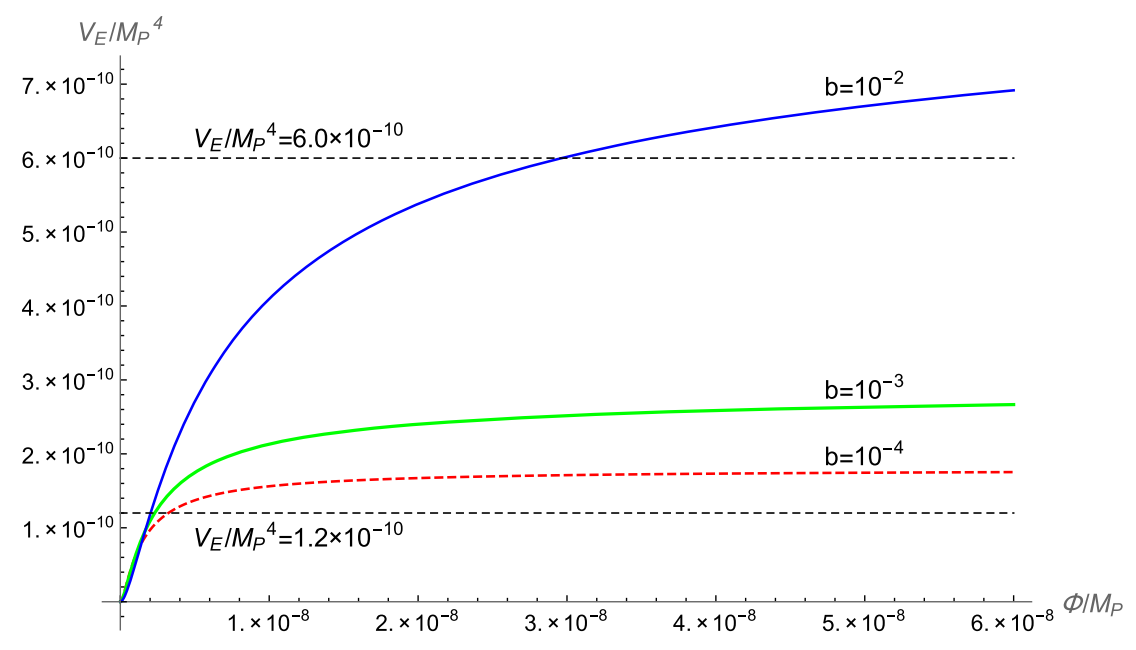

FIG. 1. The Einstein frame potential $V_{E}$ from Eq. (35) is shown for $b=10^{-2}$ (top blue curve), $b=10^{-3}$ (middle green curve) and $b=10^{-4}$ (lower red dashed curve). In this figure, $a=7 \times 10^{8}, \mu=10^{-5} H_{E}$. The upper (lower) black dashed curve shows $V_{E}=$ $6 \times 10^{-10} M_{\mathrm{P}}^{4}\left(V_{E}=1.2 \times 10^{-10} M_{\mathrm{P}}^{4}\right)$ and they represent the upper and lower limits of the effective potential corresponding to the COBE constraint (45) evaluated for $r=10^{-2}\left(r=2 \times 10^{-3}\right)$. 


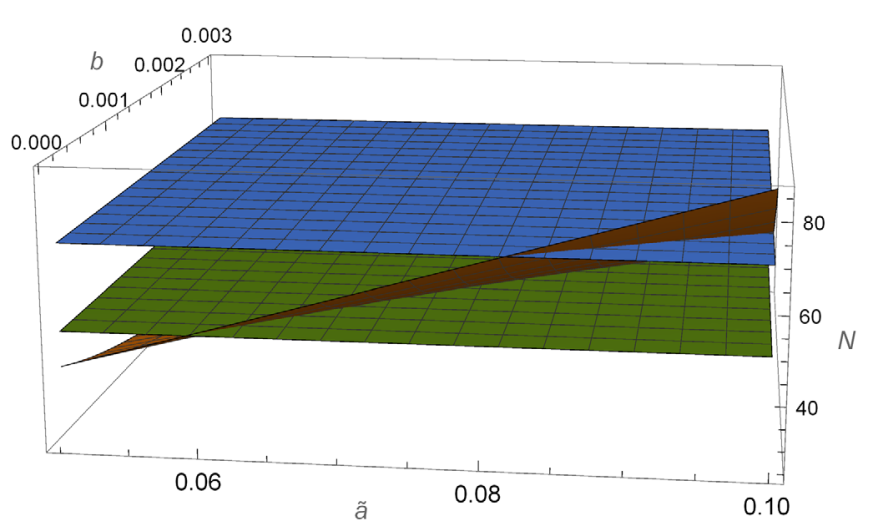

FIG. 2. The number of $e$-foldings $N$ as a function of $\tilde{a}=$ $10^{-10} a$ and $b$ ('diagonal' brown plane). The upper blue and lower green planes represent $N=50$ and $N=65$, respectively.

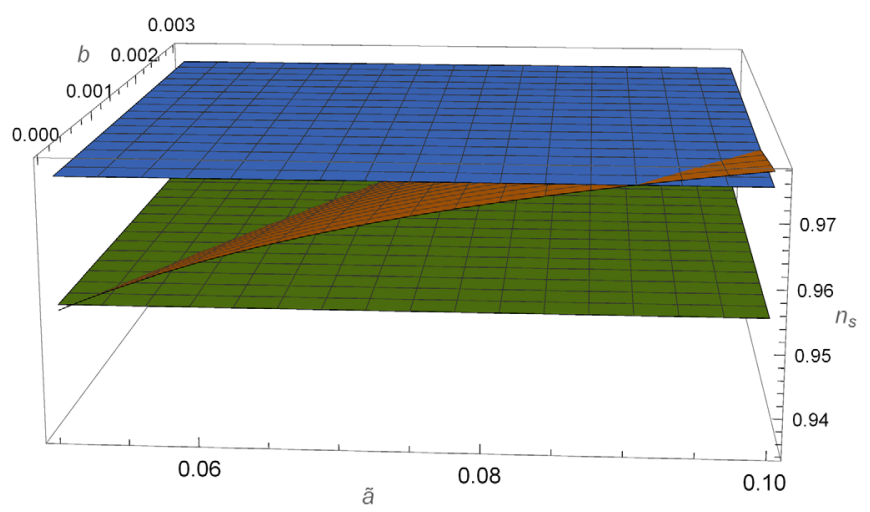

FIG. 3. The scalar spectral index $n_{s}$ as a function of parameters $\tilde{a}=10^{-10} a$ and $b$ ('diagonal' brown plane). The allowed range of $n_{s}$ is between the upper blue and lower green planes.

Next, in Fig. 3, we show typical values of $\tilde{a}$ and $b$ for which our model yields the scalar spectral index $n_{s}$ consistent with observations (the COBE constraint (45) is imposed).

From these results it follows that, when $b \geq 10^{-3}$, our inflationary model predictions depend significantly on $b$. However, when $b \ll 10^{-3}$, model predictions are to a large extent independent on $b$, and they can be well approximated by those of the Starobinsky model.

\section{RESULTS}

In this section, we present our main results; i.e., we plot the scalar spectral index $n_{s}$ (41), its running $\alpha=$ $d n_{s} / d \ln (k)$ (43) and tensor-to-scalar ratio $r$ (42) evaluated at the fiducial comoving momentum $k=k_{*}=0.05 \mathrm{Mpc}^{-1}$ (the tensor spectral index $n_{t}$ is in our model given by the consistency relation, $n_{t}=-r / 8$ and it is therefore not an independent observable). Even though it appears that $n_{s}, \alpha$ and $r$ are given in terms of the three slow roll parameters $\epsilon_{E}, \eta_{E}$ and $\xi_{E}$, they are not all independent. In fact, our

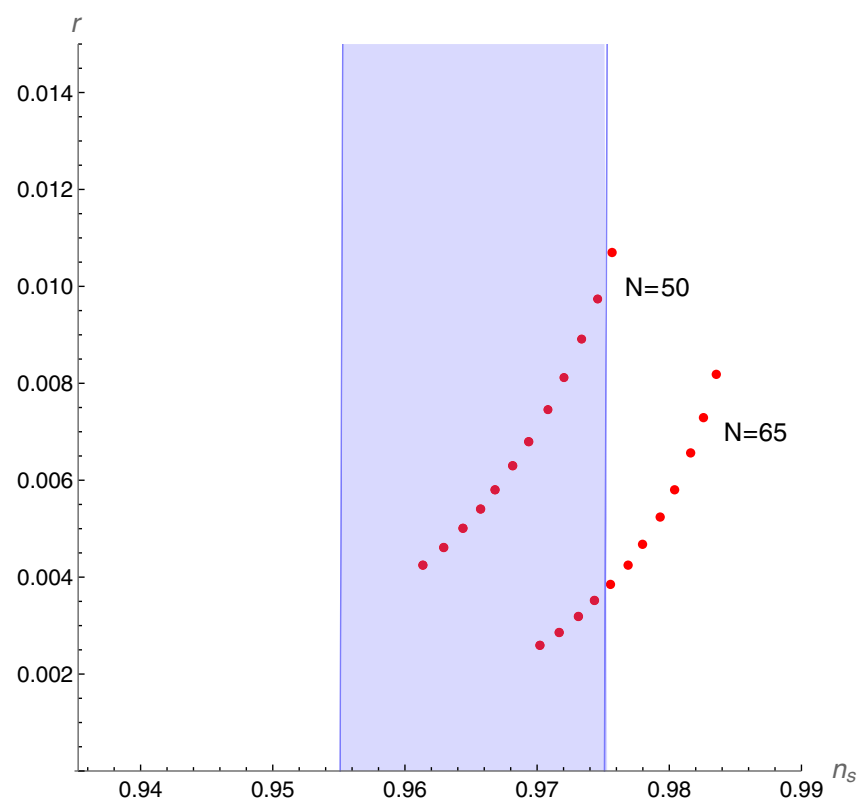

FIG. 4. The tensor-to-scalar ratio $r$ as a function of the scalar spectral index $n_{s}$ for $b$ in the range from $10^{-4}$ to $10^{-2}$. We show two curves: $N=50$ (upper red dotted curve) and $N=65$ (lower red dotted curve). The shadowed region shows the allowed values of $n_{s}$ (33). The current upper limit on the tensor-to-scalar ratio, $r<0.09$ (34) is outside the plot's range. The COBE constraint (45) is imposed and $\mu=10^{-5} H_{E}$.

model (3) is specified by two parameters, $a$ and $b$ (defined at a fiducial scale $\mu$ ), and therefore in slow roll approximation and when the COBE normalization is imposed, one free parameter remains (the number of $e$-foldings is fixed by the value of the field, $\Phi=\Phi\left(\phi_{E}\right)$ ). That means that, for any given $N$, the prediction of our model can be represented by a two-dimensional plane on the three dimensional configuration space spanned by $\left(n_{s}, r, \alpha\right)$. The constraint on $n_{s}$ is then the three dimensional region on that configuration space defined by $n_{s, \min } \simeq 0.955 \leq n_{s} \leq n_{\mathrm{s}, \max } \simeq 0.975$, see Eq. (33). Rather than showing this three-dimensional space, for the sake of clarity we show in what follows its three cross sections with the planes $\left(n_{s}, r\right),\left(n_{s}, \alpha\right)$, and $(r, \alpha)$.

In Fig. 4, we show how the tensor-to-scalar ratio $r$ depends on the scalar spectral index $n_{s}$. The upper (lower) red dotted curve shows $r=r\left(n_{s}\right)$ for $N=50(N=65), b$ ranges from $10^{-4}$ to $10^{-2}$, and $a$ is fixed by the COBE constraint. For very small values of $b$ one recovers the predictions of the $R^{2}$-model, $r \in[2,4] \times 10^{-3}$, and $n_{s}$ lies in the sweet spot of the allowed $n_{s}$. When $b$ increases, however, both $r$ and $n_{s}$ increase, such that when $r$ exceeds $r \simeq 10^{-2}, n_{s}$ becomes too large to be consistent with observations. Notice that even a modest increase in $r$ can have very beneficial consequences for detectability of tensor modes. Namely, while advanced future satellite CMB probes (such as COrE [53] and LiteBIRD [54]) can detect $r \sim 3 \times 10^{-3}$ with an accuracy of a few standard deviations, if $r \simeq 1 \times 10^{-2}$ these probes can claim 


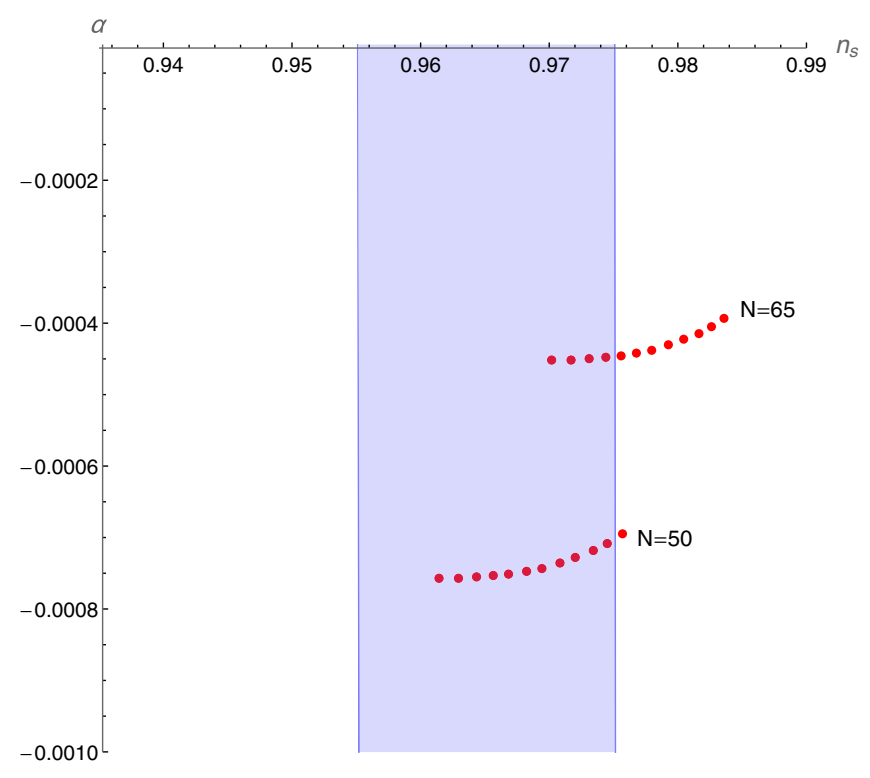

FIG. 5. The scalar spectrum index running parameter $\alpha$ as a function of the scalar spectral index $n_{s}$ for $b$ in the range from $10^{-4}$ to $10^{-2}$. We show two curves: $N=50$ (upper dotted curve) and $N=65$ (lower dotted curve). The shadowed region shows the allowed values of $n_{s}$ in Eq. (33). The current Planck Collaboration limits on the running of scalar spectral index, $\alpha \in[-0.010,0.004]$ lie outside the plot's range. The COBE constraint (45) is imposed and $\mu=10^{-5} H_{E}$.

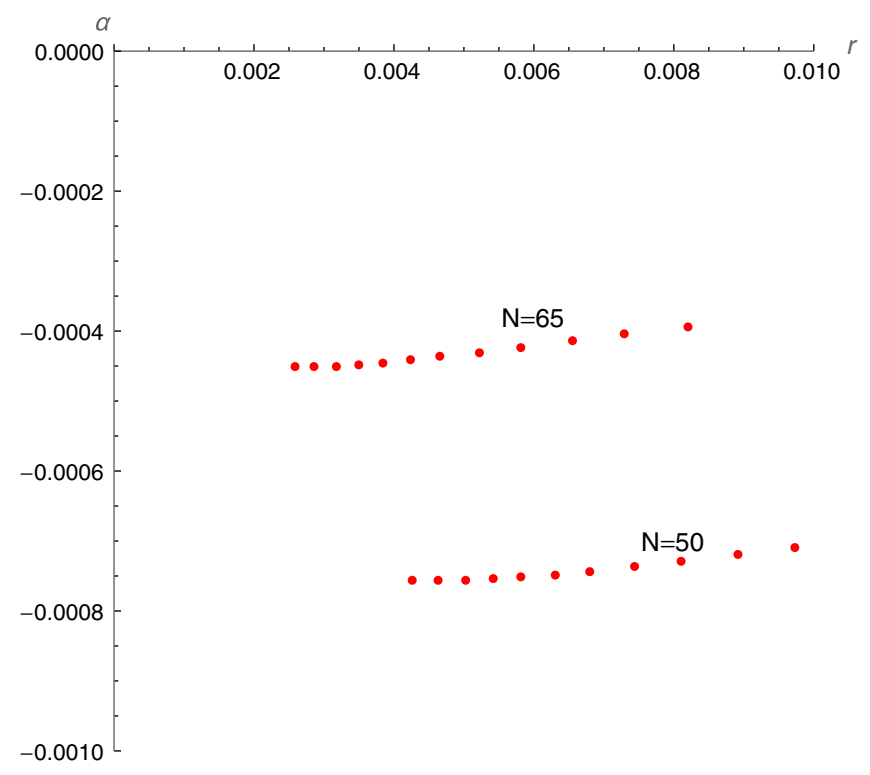

FIG. 6. The the running of scalar spectrum index $\alpha$ as a function of the tensor-to-scalar ratio $r$ for $b$ in the range from $10^{-4}$ to $10^{-2}$. We show two curves: $N=50$ (upper dotted curve) and $N=65$ (lower dotted curve). The shadowed region shows the allowed values of $n_{s}$ in Eq. (33). The current upper limit on the tensor-to-scalar ratio, $r<0.09$ (34) is outside the plot's range. The COBE constraint (45) is imposed and $\mu=10^{-5} H_{E}$. discovery (i.e., more than $5 \sigma$ detection), and moreover even Stage IV of earth-based CMB observatories can detect $r \simeq 10^{-2}$.

In Fig. 5, in which we impose identical constraints as in Fig. 4, we show $\alpha=\alpha\left(n_{s}\right)$ for $N=65$ (upper red dots) and for $N=50$ (lower red dots) with $b \in\left[10^{-4}, 10^{-2}\right]$. As in Fig. 4 , as $b$ increases, $n_{s}$ increases, eventually leaving the allowed range for $n_{s}$ when $b>10^{-2}$. Note that $\alpha$ does not depend significantly on $b$, so the logarithmic dependence $\ln \left(R / \mu^{2}\right)$ in the effective action (3) does not affect detectability of $\alpha$. In order to detect $\alpha$ in our model, we need an improvement of about 1 order of magnitude with respect to current observations, which is possible only with space based observations (such as COrE), but unlikely with earthbased measurements. Nevertheless, together with a detection of $r$, a detection of $\alpha$ would represent an important milestone in testing the model proposed in this work.

For completeness, in Fig. 6, we show $\alpha=\alpha(r)$ for $N=65$ (upper curve) and $N=50$ (lower curve). As expected, as $b$ increases from $10^{-4}$ to $10^{-2}, r$ increases (reaching $r \sim 10^{-2}$ when $b \sim 10^{-2}$ ) and $\alpha$ does not change much.

\section{CONCLUSION}

We consider an effective gravity model (3) motivated by Weinberg's proposal that gravity might be renormalizable in a weaker sense, known as asymptotic safety [16-19]. This model is supported both by perturbative studies of quantum gravity [8-11,44] as well as by more recent studies [22] based on functional renormalization group approach to quantum gravity. Our model can be considered as an improvement of Starobinsky $R^{2}$ inflation. Our main results can be stated as follows:

(i) The effective theory of gravity (3) represents a viable model of inflation, provided $a$ and $b$ are appropriately chosen, i.e., $a \sim 10^{8}-10^{9}$ (as implied by the COBE constraint) and $b \leq 10^{-2}$ (in order that the scalar spectral index $n_{s}$ agrees with observations);

(ii) When $b=0$ we recover the predictions of the Starobinsky $R^{2}$ inflationary model, which is currently a viable model of inflation and for which the tensor-to-scalar ratio is about, $r \simeq 3 \times 10^{-3}$;

(iii) As $b$ increases from $b=0$ to about $b \simeq 10^{-2}, r$ increases from about $3 \times 10^{-3}$ to about $1 \times 10^{-2}$, which is much easier to detect by future $\mathrm{CMB}$ polarization observatories, such as LiteBIRD and COrE. This increase in $r$ represents the main result of this work. Namely, observing $r$ in the range $r \in$ $[5,10] \times 10^{-3}$ (and significantly larger than the value in the Starobinsky model, $\left.r=3 \times 10^{-3}\right), n_{s} \simeq$ 0.970-0.975 (which is about one standard deviation higher than the current central value for $n_{s}$ ) and $\alpha \simeq$ $-5 \times 10^{-4}$ (and significantly different from zero) would represent observational evidence that would lend support to gravity as an asymptotically safe 
theory and that it exhibits an ultraviolet fixed point on super-Planckian energy scales. We are not aware of any other observations that could test gravity on (super-)Planckian scales. Moreover, measuring $r \sim 10^{-2}$ would allow future space missions to test the consistency relation, $r=-8 n_{t}$, which represents an important consistency test of one field inflationary models. Owing to the smallness of $r$, the consistency relation will be much harder to test in the original Starobinsky model.

At first sight, our model might seem unnatural, because the required value of $a$ is very large. However, from the observational point of view it is the observed smallness of inhomogeneous perturbations in the Universe that determines this large value unambiguously. Also, from the theoretical point of view, one should keep in mind, however, that the value of $a$ in Eq. (3) is not fixed by quantum loop effects. Indeed, adding an arbitrary finite coefficient $\Delta a$ to $a$ in (3) is consistent with quantum loop effects, i.e., one can fix $a=a(\mu)$ arbitrarily (in principle by observations) at some reference scale $\mu$, and then $a(\mu, R)$ will run according to a suitable renormalization group equation, for more details see [22]. On the other hand, once one fixes $a$, one cannot arbitrarily choose $b$; the value of $b$ depends on the bare Lagrangian, i.e., on the particle content of the theory, and for pure gravity the relation is given by (2), in which case $b \sim 10^{-4}$. It would be of interest to investigate precisely how the relation between $b$ and $a$ depends on the field content of the theory, and for what type of theories one gets the values of $n_{s}, r$ and $\alpha$ that are of interest for observational cosmologists. As a final remark, we note that measuring $b$ would provide us with some information (albeit very limited) on the structure of perturbative quantum gravity at the grand unified scale (of inflation) and (via the threshold effects) even on the physics at and beyond the Planck scale.

\section{ACKNOWLEDGMENTS}

A. S. is supported by the RSF Grant No. 16-12-10401. L. L. is funded by the Chinese Scholarschip Council (CSC). T.P. and L. L. acknowledge support from the D-ITP consortium, a program of the NWO that is funded by the Dutch Ministry of Education, Culture and Science (OCW). This work is part of the research program of the Foundation for Fundamental Research on Matter (FOM), which is part of the Netherlands Organisation for Scientific Research (NWO).

\section{APPENDIX: COSMOLOGICAL PERTURBATIONS IN THE JORDAN FRAME}

Here we present a derivation of expressions for the power spectra of scalar and tensor perturbations generated during inflation in modified $f(R)$ gravity with the action,

$$
S=\frac{1}{2} \int d^{4} x \sqrt{-g} f(R),
$$

directly in the physical (Jordan) frame, such that $f(R)=$ $M_{P}^{2} R$ in GR. It becomes simple if one uses the fact [55] that slow-roll inflation in this class of models occurs for the range of $R$ where $f(R)$ is close to $R^{2}$, more exactly, $f(R)=$ $A(R) R^{2}$ where $A(R)$ is a slowly changing function of $R$,

$$
\left|A^{\prime}(R)\right| \ll \frac{A(R)}{R}, \quad\left|A^{\prime \prime}(R)\right| \ll \frac{A(R)}{R^{2}},
$$

where a prime denotes the derivative with respect to $R$. The conditions (A2) are the analogues of the slow-roll conditions for the Einstein frame potential $V_{E}\left(\phi_{E}\right)$ flatness. They may be satisfied either over some interval of $R$ values, or even in one point $R=R_{0}$ only.

Then, from the trace equation of $f(R)$ gravity (written in the presence of matter with the energy density $\rho_{m}$ and the pressure $p_{m}$ for generality),

$$
\frac{3}{a^{3}} \frac{d}{d t}\left(a^{3} \frac{d f^{\prime}(R)}{d t}\right)-R f^{\prime}(R)+2 f(R)=\rho_{m}-3 p_{m},
$$

an expression follows for the number of $e$-folds in Jordan frame during inflation counted from its end $\left(R=R_{\text {end }}\right)$ back in time

$$
N(R)=-\frac{3}{2} \int_{R_{\text {end }}}^{R} d \tilde{R} \frac{A(\tilde{R})}{A^{\prime}(\tilde{R}) \tilde{R}^{2}} \gg 1 .
$$

Note that the condition $A^{\prime}<0$ is needed for the correct evolution during inflation and the graceful exit from it to the region of small curvature.

The power spectrum of tensor perturbations (summed over polarizations) can be directly obtained from the corresponding expression for inflation in GR first derived in [1] by the substitution $M_{\mathrm{P}}^{2} \rightarrow M_{\mathrm{P} \text {, fff }}^{2}=d f / d R \approx 2 A R$,

$$
\Delta_{t}^{2}(k)=\frac{1}{12 A\left(R_{k}\right) \pi^{2}},
$$

where, as usually, the index $k$ means that the corresponding quantity is estimated at the moment $t=t_{k}$ when each spatial Fourier mode of perturbations crosses the Hubble radius during inflation: $k=a\left(t_{k}\right) H\left(t_{k}\right)$. Note, however, that $d f / d R$ has to be calculated with better accuracy in order to find the correct value of the slope $n_{t}$ of the tensor spectrum.

Thus, at present $N(k)=\ln \left(k_{\text {end }} / k\right)$ where $k_{\text {end }}$ is the comoving wave vector of perturbations which crossed the Hubble radius at the end of inflation. $k_{\text {end }} / a_{0}$ [where $a_{0}$ is the present value of the scale factor $a(t)]$ is a few orders of magnitude smaller than the present CMB temperature $T_{\gamma}$. 
Its exact value depends, in particular, on duration of the epoch of the scalaron decay and creation and heating of matter after inflation.

The easiest way to find the scalar perturbation spectrum is to use the $\delta N$ formalism first introduced in [56] (and even in the fully nonlinear regime) according to which spatial inhomogeneity of total number of $e$-folds during inflation leads to the following value of the scalar perturbation $\mathcal{R}$ :

$$
\mathcal{R}(\mathbf{r})=\delta N_{\text {tot }}(\mathbf{r})=\frac{d N(R)}{d R} \delta R(\mathbf{r}),
$$

where $\delta R$ is estimated at the characteristic time of its Hubble radius crossing during inflation. This method was applied, in particular, in [57] where the quantitatively correct expressions for the power spectra of scalar and tensor perturbations in the Starobinsky model were first obtained, see also [58] and numerous later papers. ${ }^{2}$ Using (A4) and the correctly normalized rms value of $\delta R$ fluctuations, we get

$\Delta_{\mathcal{R}}^{2}(k)=\frac{A\left(R_{k}\right)}{64 \pi^{2} A^{\prime}\left(R_{k}\right)^{2} R_{k}^{2}}=-\left(96 \pi^{2} \frac{d A(R(N))}{d N}\right)^{-1}$.

Thus,

$$
r \equiv \frac{\Delta_{t}^{2}(k)}{\Delta_{\mathcal{R}}^{2}(k)}=\frac{16 A^{\prime}\left(R_{k}\right)^{2} R_{k}^{2}}{3 A\left(R_{k}\right)^{2}}
$$

and it can be checked that $r=-8 n_{t}$. For general inflationary models in $f(R)$ gravity, the power spectrum of scalar perturbations was derived in [60].

For the Starobinsky model, $A(R)=M_{\mathrm{P}}^{2}\left(\frac{1}{R}+\frac{1}{6 M^{2}}\right)$ where $M$ is the scalaron mass after the end of inflation (in particular, in flat spacetime). The first term in $A$ is much less than the second one during inflation, so it may be neglected in $A$ itself, but not in its derivative $A^{\prime}$. So,

\footnotetext{
${ }^{2}$ The correct estimate of the slope of the scalar power spectrum $n_{s}$ in this model was found even earlier in [59].
}

$$
\begin{gathered}
R(N)=4 N M^{2}, \quad \Delta_{\mathcal{R}}^{2}(k)=\frac{M^{2} N^{2}(k)}{24 \pi^{2} M_{P}^{2}}, \\
n_{s}=1-\frac{2}{N(k)}, \quad r=\frac{12}{N^{2}(k)} .
\end{gathered}
$$

In our model,

$$
A=\frac{M_{P}^{2}}{R}+\frac{a}{1+b \ln \left(R / \mu^{2}\right)}
$$

with $a \gg 1$. Using Eq. (A4), we get with sufficient accuracy:

$N(R)=\frac{3 a}{2} \int_{R_{\text {end }}}^{R} d \tilde{R} \frac{1+b \ln \frac{\tilde{R}}{\mu^{2}}}{M_{P}^{2}\left[1+b \ln \left(\tilde{R} / \mu^{2}\right)\right]^{2}+a b \tilde{R}}$.

Let us assume that the logarithmic correction is small, $b \ll 1, b \ln \left(R / \mu^{2}\right) \ll 1$. Then Eq. (A11) can be integrated analytically and

$$
1+\frac{a b R}{M_{P}^{2}}=\exp \left(\frac{2 b N}{3}\right)
$$

If $b N \ll 1$, then $a b R \ll M_{P}^{2}$ and we return to the results (A9) for the Starobinsky model with $a=M_{P}^{2} / 6 M^{2}$. In the opposite limit $b N \gg 1, a b R \gg M_{P}^{2}$ (but still $R \ll M_{P}^{2}$ that requires $a b \gg 1$ ),

$$
\begin{aligned}
& R=\frac{M_{P}^{2}}{a b} \exp \left(\frac{2 b N}{3}\right), \quad \Delta_{\mathcal{R}}^{2}(k)=\frac{1}{64 \pi^{2} a b^{2}}, \\
& n_{s} \approx 1, \quad r=\frac{16 b^{2}}{3} .
\end{aligned}
$$

Thus, it may not be reached for the observable range of perturbation wavelengths due to a too large value of $n_{s} \approx 1$ which is independent both of $k$ and of the model parameters $a, b$. These analytical results help to understand the numerical results of Sec. III.
[1] A. A. Starobinsky, Spectrum of relict gravitational radiation and the early state of the universe, Pis'ma Zh. Eksp. Teor. Fiz. 30, 719 (1979) [JETP Lett. 30, 682 (1979)].

[2] A. A. Starobinsky, A new type of isotropic cosmological models without singularity, Phys. Lett. B 91, 99 (1980).

[3] A. H. Guth, The inflationary universe: A possible solution to the horizon and flatness problems, Phys. Rev. D 23, 347 (1981).

[4] A. D. Linde, A new inflationary universe scenario: A possible solution of the horizon, flatness, homogeneity, isotropy and primordial monopole problems, Phys. Lett. B 108, 389 (1982).

[5] J.F. Donoghue, General relativity as an effective field theory: The leading quantum corrections, Phys. Rev. D 50, 3874 (1994).

[6] P. A. R. Ade et al. (Planck Collaboration), Planck 2015 results. XX. Constraints on inflation, Astron. Astrophys. 594, A20 (2016).

[7] A. A. Starobinsky, Nonsingular model of the Universe with the quantum-gravitational de Sitter stage and its 
observational consequences, in Proceedings of the Second Seminar, Quantum Theory of Gravity (Moscow, 1981) (INR Press, Moscow, 1982), p. 58; reprinted in Quantum Gravity, edited by M. A. Markov and P. C. West (Plenum, New York, 1984), p. 103.

[8] K. P. Stanyukovich, Gravitational Field and Elementary Particles [(Gravitatsionnoie pole i elementarnyie chastitsy] (Nauka, Moscow, 1965).

[9] B.S. DeWitt, Quantum theory of gravity. 1. The canonical theory, Phys. Rev. 160, 1113 (1967).

[10] B. S. DeWitt, Quantum theory of gravity. 3. Applications of the covariant theory, Phys. Rev. 162, 1239 (1967).

[11] V. L. Ginzburg, D. A. Kirzhnits, and A. A. Lyubushin, The role of quantum fluctuations of the gravitational field in general relativity and cosmology, Zh. Eksp. Teor. Fiz. 60, 451 (1971) [Sov. Phys. JETP 33, 242 (1971)].

[12] Ya. B. Zeldovich and A. A. Starobinsky, Particle production and vacuum polarization in an anisotropic gravitational field, Zh. Eksp. Teor. Fiz. 61, 2161 (1971) [Sov. Phys. JETP 34, 1159 (1972)].

[13] G. 't Hooft and M. J. G. Veltman, One loop divergences in the theory of gravitation, Ann. Inst. Henri Poincaré, A 20, 69 (1974).

[14] M. H. Goroff and A. Sagnotti, Quantum gravity at two loops, Phys. Lett. B 160, 81 (1985).

[15] A. E. M. van de Ven, Two loop quantum gravity, Nucl. Phys. B378, 309 (1992).

[16] S. Weinberg, Critical Phenomena for Field Theorists (Spinger, New Yorlk, 1979).

[17] S. Weinberg, in General Relativity: An Einstein Centenary Survey, edited by S. W Hawking and W. Israel (Cambridge University Press, Cambridge, England, 1979), p. 790.

[18] S. Weinberg, Effective field theory, past and future, Int. J. Mod. Phys. A 31, 1630007 (2016).

[19] S. Weinberg, Effective field theory, past and future, Proc. Sci., CD09 (2009) 001, arXiv:0908.1964.

[20] M. Reuter, Nonperturbative evolution equation for quantum gravity, Phys. Rev. D 57, 971 (1998).

[21] M. Reuter and F. Saueressig, Quantum Einstein gravity, New J. Phys. 14, 055022 (2012).

[22] M. Demmel, F. Saueressig, and O. Zanusso, A proper fixed functional for four-dimensional quantum Einstein gravity, J. High Energy Phys. 08 (2015) 113.

[23] C. Fang and Q. G. Huang, The trouble with asymptotically safe inflation, Eur. Phys. J. C 73, 2401 (2013).

[24] Q.-G. Huang, A polynomial $f(R)$ inflation model, J. Cosmol. Astropart. Phys. 02 (2014) 035.

[25] A. Marunović and T. Prokopec, Planck scale operators, inflation and fine tuning, Phys. Rev. D 96, 043512 (2017).

[26] M. Reuter and F. Saueressig, From big bang to asymptotic de Sitter: Complete cosmologies in a quantum gravity framework, J. Cosmol. Astropart. Phys. 09 (2005) 012.

[27] A. Bonanno and M. Reuter, Cosmology of the Planck era from a renormalization group for quantum gravity, Phys. Rev. D 65, 043508 (2002).

[28] A. Bonanno and M. Reuter, Cosmology with self-adjusting vacuum energy density from a renormalization group fixed point, Phys. Lett. B 527, 9 (2002).
[29] A. Bonanno, A. Contillo, and R. Percacci, Inflationary solutions in asymptotically safe $\mathrm{f}(\mathrm{R})$ theories, Classical Quantum Gravity 28, 145026 (2011).

[30] K. Falls, D. F. Litim, K. Nikolakopoulos, and C. Rahmede, On de Sitter solutions in asymptotically safe $f(R)$ theories, Classical Quantum Gravity 35, 135006 (2018).

[31] S. Weinberg, Asymptotically safe inflation, Phys. Rev. D 81, 083535 (2010).

[32] S.-H. H. Tye and J. Xu, Comment on asymptotically safe inflation, Phys. Rev. D 82, 127302 (2010).

[33] M. Hindmarsh, D. Litim, and C. Rahmede, Asymptotically safe cosmology, J. Cosmol. Astropart. Phys. 07 (2011) 019.

[34] A. Bonanno and F. Saueressig, Asymptotically safe cosmology-A status report, C.R. Phys. 18, 254 (2017).

[35] Y. F. Cai and D. A. Easson, Asymptotically safe gravity as a scalar-tensor theory and its cosmological implications, Phys. Rev. D 84, 103502 (2011).

[36] E. J. Copeland, C. Rahmede, and I. D. Saltas, Asymptotically safe Starobinsky inflation, Phys. Rev. D 91, 103530 (2015).

[37] A. Codello, J. Joergensen, F. Sannino, and O. Svendsen, Marginally deformed Starobinsky gravity, J. High Energy Phys. 02 (2015) 50.

[38] K. Bamba, G. Cognola, S. D. Odintsov, and S. Zerbini, Oneloop modified gravity in a de Sitter universe, quantumcorrected inflation, and its confrontation with the Planck result, Phys. Rev. D 90, 023525 (2014).

[39] I. Ben-Dayan, S. Jing, M. Torabian, A. Westfal, and L. Zarate, $R^{2} \log R$ quantum corrections and the inflationary observables, J. Cosmol. Astropart. Phys. 09 (2014) 005.

[40] A. Bonanno and A. Platania, Asymptotically safe inflation from quadratic gravity, Phys. Lett. B 750, 638 (2015).

[41] M. Rinaldi, G. Cognola, L. Vanzo, and S. Zerbini, Reconstructing the inflationary $f(R)$ from observations, J. Cosmol. Astropart. Phys. 08 (2014) 015.

[42] S. R. Coleman and E. J. Weinberg, Radiative corrections as the origin of spontaneous symmetry breaking, Phys. Rev. D 7, 1888 (1973).

[43] N. Alkofer and F. Saueressig, Asymptotically safe $f(R)$ gravity coupled to matter I: the polynomial case, arXiv: 1802.00498.

[44] V. T. Gurovich and A. A. Starobinsky, Quantum effects and regular cosmological models, Sov. Phys. JETP 50, 844 (1979).

[45] G. K. Chakravarti and S. Mohanty, Power law Starobinsky model of inflation from no-scale SUGRA, Phys. Lett. B 746, 242 (2015).

[46] H. Motohashi, Consistency relation for $R^{p}$ inflation, Phys. Rev. D 91, 064016 (2015).

[47] L. H. Liu, Analysis of $R^{p}$ inflationary model as $p \geq 2$, arXiv:1807.00666.

[48] M. Rinaldi, G. Cognola, L. Vanzo, and S. Zerbini, Inflation in scale-invariant theories of gravity, Phys. Rev. D 91, 123527 (2015).

[49] N. Palanque-Delabrouille et al., Cosmology with Lymanalpha forest power spectrum, J. Cosmol. Astropart. Phys. 11 (2015) 011.

[50] P. A. R. Ade et al. (BICEP2 and Planck Collaborations), Joint Analysis of BICEP2/KeckArray and Planck Data, Phys. Rev. Lett. 114, 101301 (2015). 
[51] P. A. R. Ade et al. (BICEP2 and Keck Array Collaborations), Improved Constraints on Cosmology and Foregrounds from BICEP2 and Keck Array Cosmic Microwave Background Data with Inclusion of $95 \mathrm{GHz}$ Band, Phys. Rev. Lett. 116, 031302 (2016).

[52] Y. Akrami et al. (Planck Collaboration), Planck 2018 results. X. Constraints on inflation, arXiv:1807.06211.

[53] F. R. Bouchet et al. (COrE Collaboration), COrE (Cosmic Origins Explorer) A White Paper, arXiv: 1102.2181.

[54] T. Matsumura et al., Mission design of LiteBIRD, J. Low Temp. Phys. 176, 733 (2014).

[55] S. A. Appleby, R. A. Battye, and A. A. Starobinsky, Curing singularities in cosmological evolution of $F(R)$ gravity, J. Cosmol. Astropart. Phys. 06 (2010) 005.
[56] A. A. Starobinsky, Dynamics of phase transition in the new inflationary Universe scenario and generation of perturbations, Phys. Lett. B 117, 175 (1982).

[57] A. A. Starobinsky, The perturbation spectrum evolving from a nonsingular, initially de Sitter cosmology, and the microwave background anisotropy, Pisma v Astron. Zh. 9, 579 (1983) [Sov. Astron. Lett. 9, 302 (1983)].

[58] L. A. Kofman and V. F. Mukhanov, Evolution of perturbations in an inflationary universe, Pis'ma Zh. Eksp. Teor. Fiz. 44, 481 (1986) [JETP Lett. 44, 619 (1986)].

[59] V. F. Mukhanov and G. V. Chibisov, Quantum fluctuations and a non-singular universe, Pis'ma Zh. Eksp. Teor. Fiz. 33, 549 (1981) [JETP Lett. 33, 532 (1981)].

[60] J.-C. Hwang and H. Noh, Cosmological perturbations in generalized gravity theories, Phys. Rev. D 54, 1460 (1996). 